\title{
Design and Construction of a W-band Sheet Beam Klystron
}

\author{
G. Scheitrum \\ Stanford Linear Accelerator Center \\ 2575 Sand Hill Road \\ Menlo Park, CA 94025
}

\begin{abstract}
The design and construction of a $100 \mathrm{~kW}$ peak power, 2\% duty, PCM focused, Wband sheet beam klystron is discussed. The elliptical cross section beam is produced by a new electron gun design using a cylindrical cathode and a racetrack shaped focus electrode. The multi-gap cavities produce acceptable values of $\mathrm{R} / \mathrm{Q}$ and are designed to produce a uniform electric field over the width of the 12:1 aspect ratio beam. The prototype cavities are produced using normal machining however, LIGA will be used to fabricate the cavities in production versions.
\end{abstract}

Keywords: sheet beam klystron.

PACS: 84.40.Fe

\section{INTRODUCTION}

The W-band sheet beam klystron being developed at SLAC is intended to address several needs. First, the development of RF sources for accelerators has advanced to the point where further gains in peak power, average power or lifetime require a significant reduction in beam power density. Second, at millimeter wavelengths, there is a growing need for a high average power RF source in a reasonable size and weight package. The sheet beam klystron is a narrowband device that is ideally suited to both of these needs. The operating frequency of $95 \mathrm{GHz}$ results in a reasonably small inexpensive test vehicle that will validate the technical issues associated with operation of a PCM focused sheet beam klystron. These issues include beam formation, beam transport, cavity design, thermal capacity and device fabrication issues. Each of these areas will be discussed in turn. 


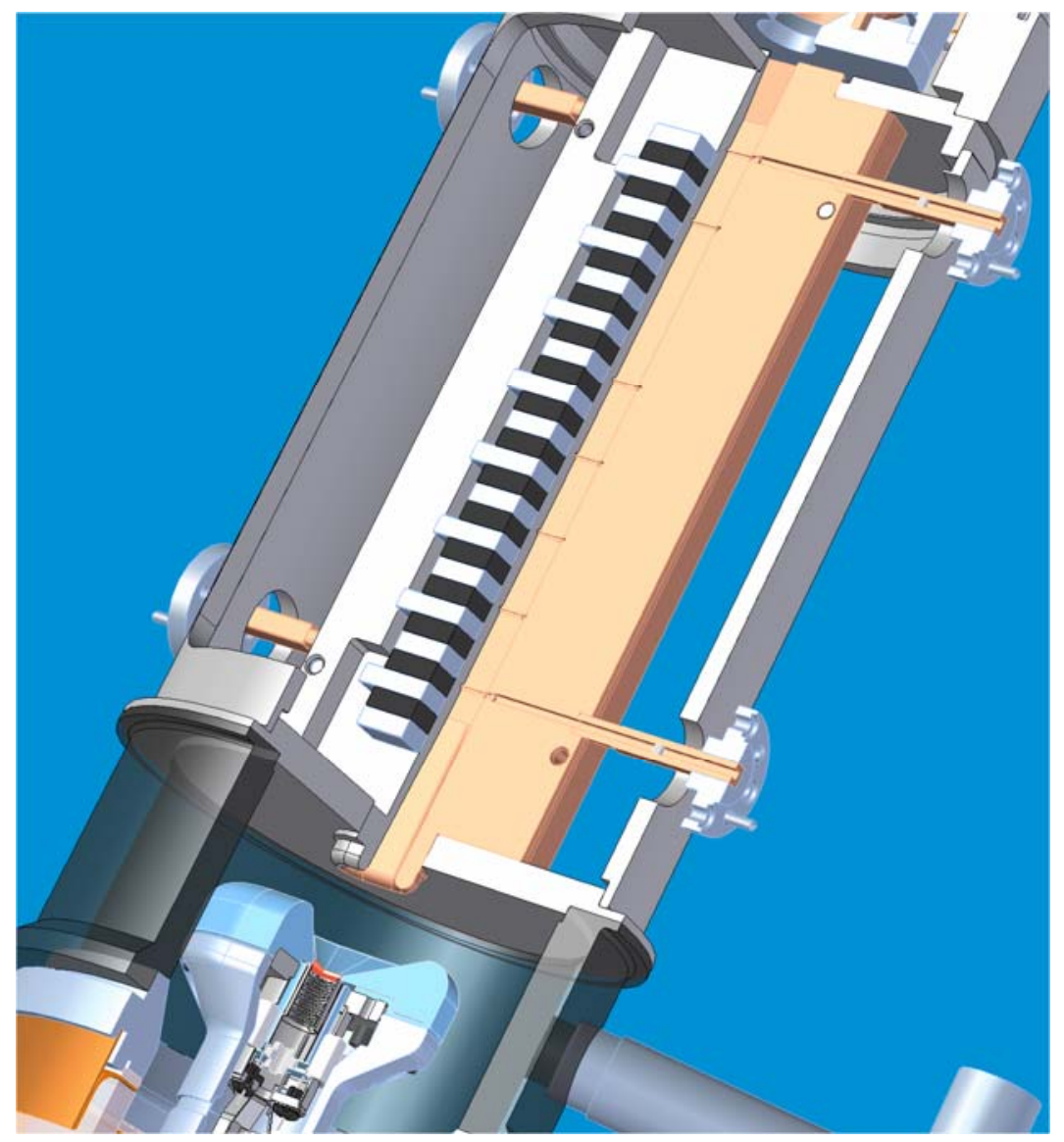

FIGURE 1. Solid model showing cutaway view of electron gun, PCM magnetic circuit and the seven cavity RF circuit of the W-band Sheet Beam Klystron.

WSBK Design Parameters

$\begin{array}{ll}\text { Beam voltage } & 74 \mathrm{kV} \\ \text { Beam current } & 3.6 \mathrm{~A}(0.18 \mu \mathrm{P}) \\ \text { Peak power } & 100 \mathrm{~kW} \\ \text { Average power } & 2 \mathrm{~kW}(2 \% \text { duty) } \\ \text { Efficiency } & 40 \% \\ \text { Gain } & 40 \mathrm{~dB} \\ \text { Brillouin Magnetic field } & 1000 \text { Gauss RMS } \\ \text { Number of cavities } & 7 \\ \text { Output cavity } & 7 \text { gap output } \\ \text { Circuit length (wg to wg) } & 9 \mathrm{~cm}\end{array}$




\section{ELECTRON BEAM FORMATION}

The initial efforts to produce an electron gun for SLAC's sheet beam klystrons focused on rectangular cathodes in focus electrodes that looked somewhat like rectangular ashtrays. The focus electrode corners were relieved to keep from overfocusing the corners of the rectangular beam. During the investigation of the optimum beam transport scheme, an elliptical beam was found to maintain its shape through the focusing system while a rectangular beam would tend to an elliptical shape with the corners of the beam being forced to the walls of the drift tube.

R. Phillips proposed a new gun design that used a cylindrical cathode with a cylindrical cut perpendicular to the beam direction. A racetrack shaped focus electrode produced focusing in the vertical plane and only a small amount of convergence in the horizontal plane. Simulations using MICHELLE show a well formed $6 \mathrm{~mm} \times 0.5 \mathrm{~mm}$ elliptical beam with an area convergence of 13.5 and a cathode to beam minimum distance of $4 \mathrm{~cm}$.
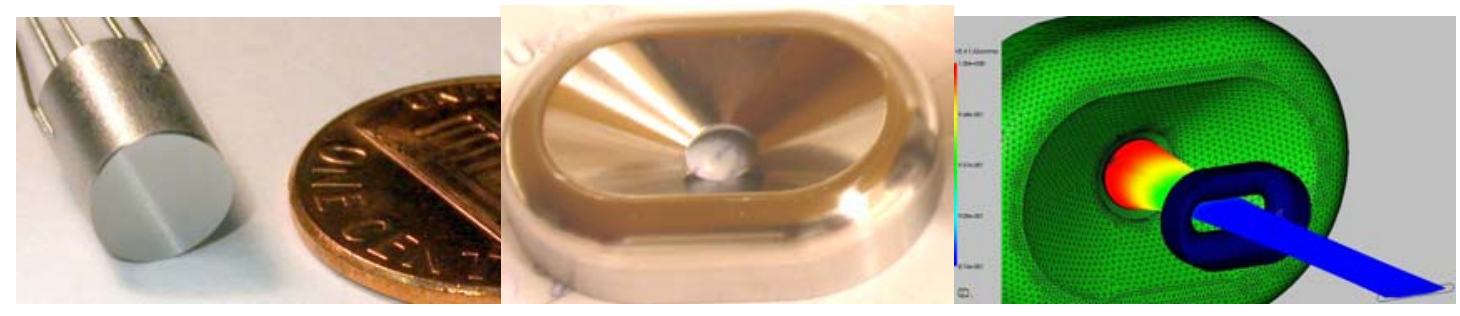

FIGURE 2. Photos of WSBK cathode and focus electrode with MICHELLE simulation output plot.

\section{ELECTRON BEAM TRANSPORT}

Permanent magnet focusing was chosen for the beam transport system for obvious reasons. Periodic cusp magnets (PCM) and wiggler focusing were evaluated and PCM was chosen because transverse excursions of the beam were larger with wiggler focusing. Focusing in the wide beam direction was accomplished using offset polepieces after Booske ${ }^{1}$ and Basten ${ }^{2}$.

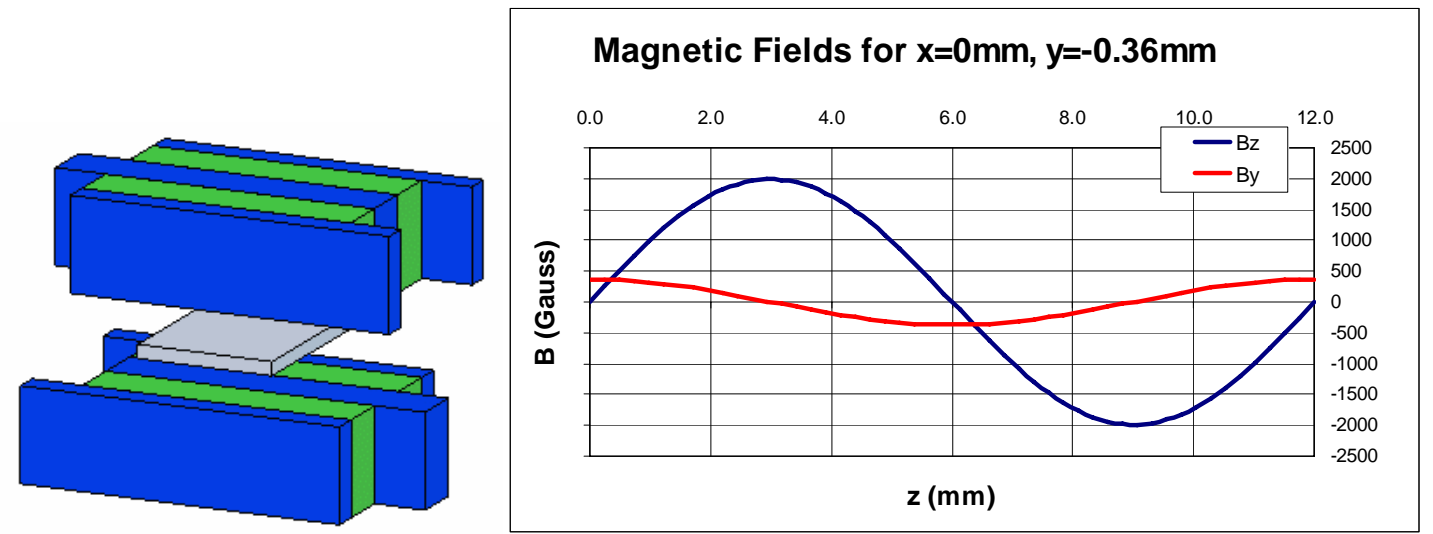

FIGURE 3. Schematic of offset polepiece PCM focusing circuit and plot of Bz and By at lower edge of drift tube $(\mathrm{y}=-0.36 \mathrm{~mm})$. 


\section{CAVITY DESIGN}

The cavities for the sheet beam klystron are based on early work at SLAC by Wilson $^{3}$ and $\mathrm{Yu}$. Cutoff waveguide sections are used to maintain a constant $\mathrm{E}_{\mathrm{z}}$ field across the width of the beam. Both cavity impedance (R/Q) and coupling factor (M) are lower in sheet beam cavities than in equivalent round beam cavities. This explains the need for intermediate and output cavities with multiple gaps. In the output cavity, seven gaps are needed to increase the total $R / Q$ and lower the required $Q_{e}$ so that the circuit efficiency is above $75 \%$.

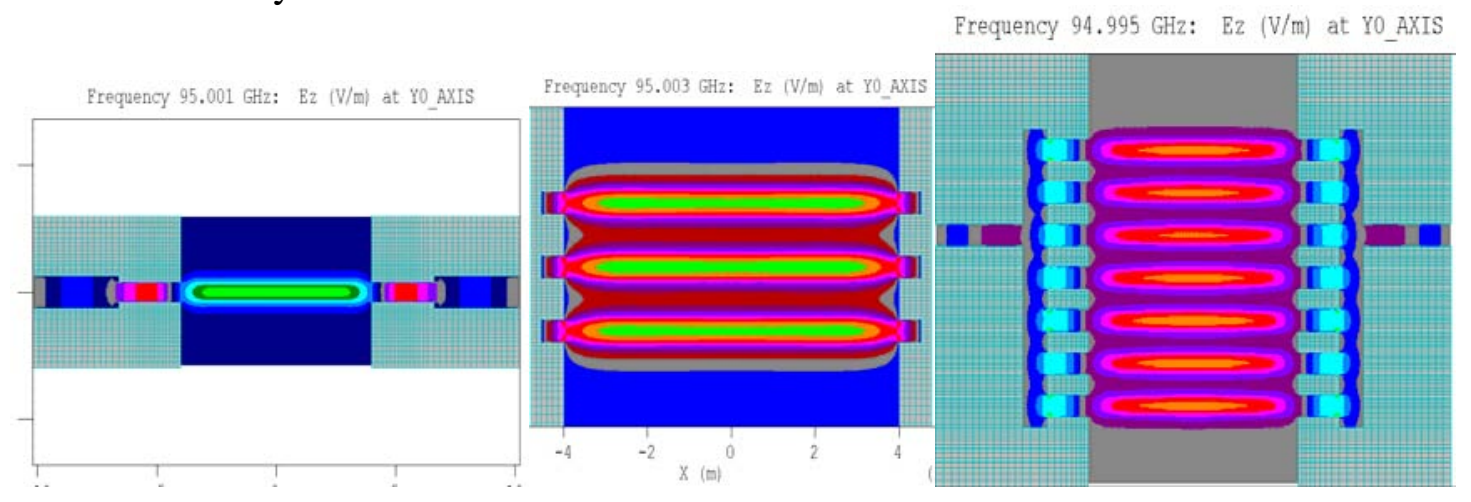

FIGURE 4. MAGIC Ez field contour plots for one, three and seven gap sheet beam cavities.

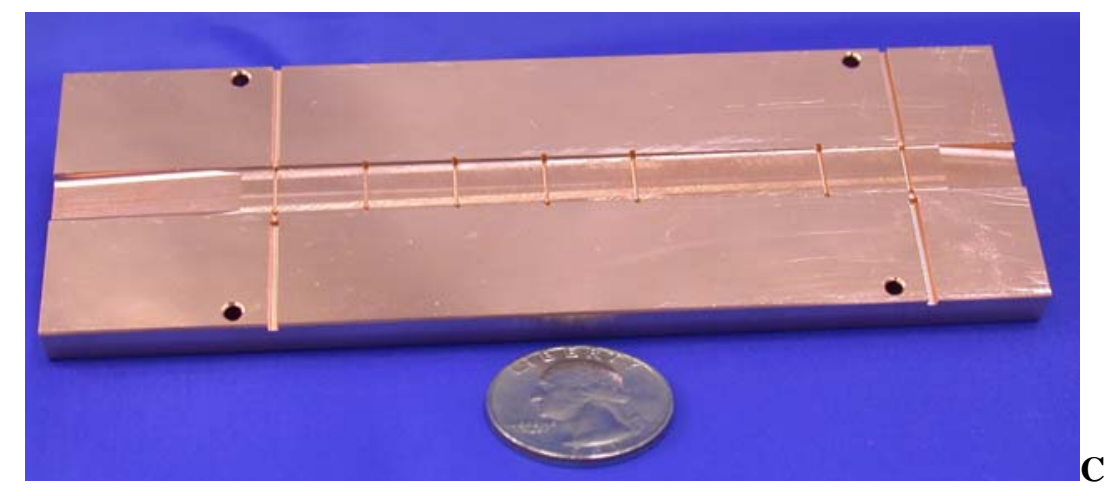

FIGURE 5. Photograph of one half of a seven cavity single-gap circuit for the WSBK. This circuit is being used in the prototype device to evaluate beam transport and small signal behavior.

\section{THERMAL ANALYSIS}

The major benefit of sheet beam RF sources is the significant increase in surface area in the RF circuit. The 12:1 beam aspect ratio results in five times the surface area in output cavity compared to a cylindrical cavity. This leads to improved heat transfer and reduces the pulse temperature rise by a factor of five. ANSYS thermal simulations using a $10 \mu$ s pulse, $2 \%$ duty, and $5 \%$ beam interception predict a pulse temperature rise of $18^{\circ} \mathrm{C}$ which is well below the plastic deformation limit for pure copper at $40^{\circ} \mathrm{C}$. 

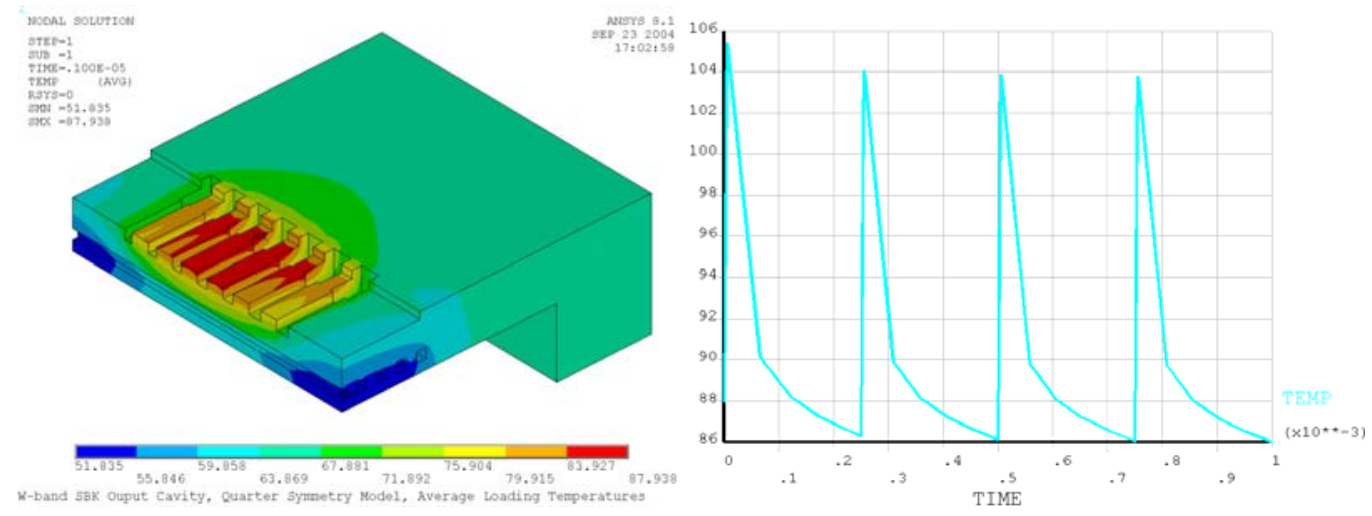

FIGURE 6. ANSYS thermal model of seven-gap WSBK output cavity.

\section{FABRICATION ISSUES}

Circuit fabrication at W-band frequencies requires dimensional accuracy on the order of one or two microns to produce structures that do not require tuning. In addition, the cavity surfaces must have finishes better than $200 \mathrm{~nm}$ to reach the cavity Q's required for good circuit efficiency. LIGA is the ideal process to produce such structures but it requires very long lead times and access to synchrotron light sources.

For the prototype sheet beam klystron, normal machining was used to reduce development time. Normal machining with high speed spindles can achieve five micron dimensional accuracy and has achieved intrinsic Q's close to the theoretical maximum for perfectly flat surfaces. Several individual multi-gap cavities and complete klystron circuits have been fabricated using high speed milling machines. The results have been surprisingly good. The variability in resonant frequencies can be accommodated by tuning the cavities after fabrication. Since the tuning rate for vertical compression is $15 \mathrm{MHz} /$ micron, cavity frequencies can be increased up to $400 \mathrm{MHz}$ with very little change in drift tube height.

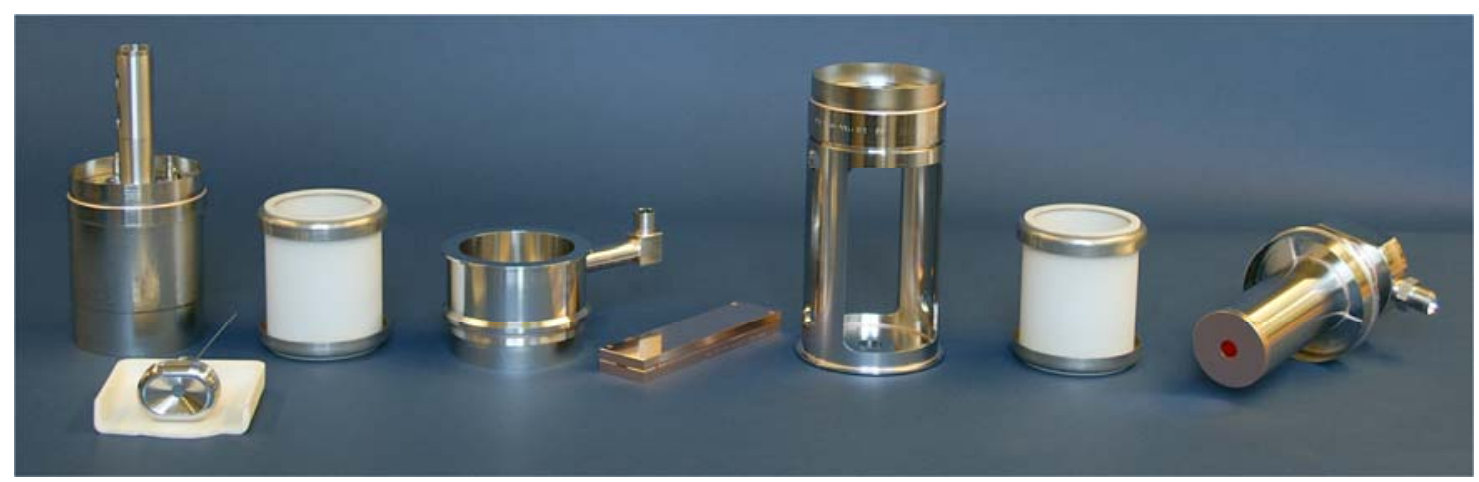

FIGURE 7. Photograph of the assemblies for the WSBK. The diffusion bonded copper circuit needs some final machining before the tube is assembled.

The gun has been modeled extensively and all electron gun to circuit alignments are relatively insensitive with the exception of the vertical alignment of the focus 
electrode and cathode to the beam aperture in the anode. This dimension requires positional accuracy of 25 to 50 microns to prevent beam interception on the anode. A new anode design is being developed to reduce the alignment sensitivity in this area.

\section{SUMMARY}

The prototype version of SLAC's W-band Sheet Beam Klystron is nearing completion. The WSBK should be on test in September. RF testing of the device should answer serious questions about beam formation, beam transport in the presence of RF fields, the mode competition issues raised with a propagating drift tube (TE modes), electronic efficiency and circuit efficiency. Successful answers to these questions should lead to multiple versions of SLAC's sheet beam klystron at frequencies from L-band to Ka-band to W-band.

\section{ACKNOWLEDGMENTS}

Work supported by both the U.S. Department of Energy under contract number DEAC02-76SF00515 and by AFOSR MURI contract 99RA0734-04

\section{REFERENCES}

1. J.H. Booske, B.D. McVey, T.M. Antonsen, JAP, 73, 4140 (1993)

2. M. A. Basten and J. H. Booske, JAP, 85, 6313 (1999)

3. D. Yu, P. Wilson, Particle Accelerator Conference, 1993., 17-20 May 1993 Page(s):2681 - 2683 vol.4 